PERIODICALS OF ENGINEERING AND NATURAL SCIENCES

Vol. 1 No. 1 (2013)

Available online at: http://pen.ius.edu.ba

\title{
The Investigation of Using For Gasoline Atomization of Ultrasonic Sound Generated By Piezoelectric Ceramic
}

\author{
Burak TANYERI \\ Firat University, Elazığ \\ TURKEY
}

\author{
Cengiz ÖNER \\ Firat University, Elazı̆ \\ TURKEY
}

\begin{abstract}
Fuel atomization is important for internal combustion engine. Therefore in this study used ultrasonic atomization method that is a new atomization method. The generated of ultrasonic sound, using field and properties, especially cavitation effect on liquids scanned in literature. Ultrasonic atomization method was experimentally compared with the existing injector atomization. Scaled droplets occurred as a result of atomization. Image processing method was used for droplet size.
\end{abstract}

As a result experimental findings transformed into image and graphics. Pictures and graphics were interpreted and written to paper.

Keywords: İnjector, ultrasonic atomization, cavitation, image processing method, droplet size.

\section{Introduction}

With all efforts to find new sources of energy, burning is, still, the main way to generate energy. This justifies the specialists studies focused on improving combustion systems. They must achieve a more complete combustion, a higher efficiency, and also have minimized pollutant emissions [1]. The atomization is very important to fuel combustion because it is effect on combustion efficiency. For this reason there are main methods of liquid fuel atomization.

One of them, which is using internal combustion engine, is mechanical spraying by pressure. In this method, a higher-pressure pump is needed because atomization is through higher-pressure. This is disadvantage.

One latter method, which is using ultrasonic nebulizer device, ultrasonic cleaner device and ultrasonic emulsion, is ultrasonic cavitation method. In this method, ultrasonic sound generated by piezoelectric ceramic that is the crystal salt. Ultrasonic cavitation is the formation of a gas bubble in the liquid during the rarefaction cycle. During the collapse tremendous pressures are produced. The pressure may be of the order of several thousand atmospheres. Thousands of these small bubbles are formed in a small volume of the liquid.

\subsection{Ultrasonic Waves and Properties}

A zone of compression and an adjacent zone of rarefaction constitute one cycle of an ultrasound wave. A wave cycle can be represented as a Fig. 1 of local pressure (particle density) in the medium versus distance in the direction of the ultrasound wav. The distance covered by one cycle is the wavelength of the ultrasound wave. The number of cycles per unit time (cps, or just sec-1) introduced into the medium each second is referred to as the frequency of the wave, expressed in units of hertz, kilohertz, or megahertz where $1 \mathrm{~Hz}$ equals $1 \mathrm{cps}$. The maximum height of the wave cycle is the amplitude of the ultrasound wave.

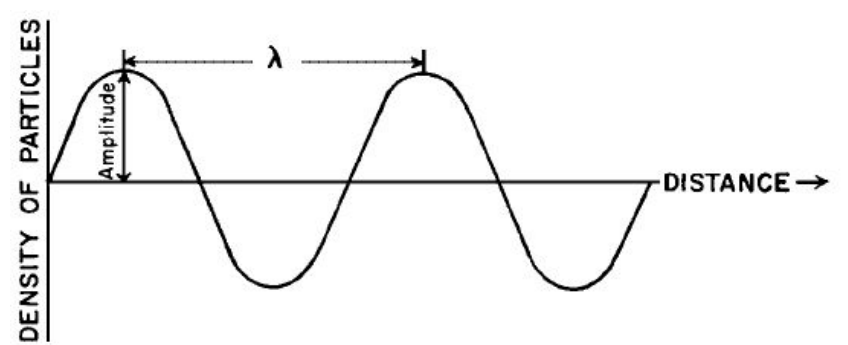

Figure.1 Characteristic of an Ultrasound Wave

Table 1. Table1. Frequency Classification of Ultrasound

\begin{tabular}{|c|c|}
\hline Frequency $(\mathrm{Hz})$ & Classification \\
\hline $20-20,000$ & Audible sound \\
\hline $20,000-1,000,000$ & Ultrasound \\
\hline $1,000,000-30,000,000$ & $\begin{array}{c}\text { Diagnostic Medical } \\
\text { Ultrasound }\end{array}$ \\
\hline
\end{tabular}

\subsection{Velocity of Ultrasound}

The velocity of spread sound depends on two importance parameter in medium. One of them is stiffness of the material constituent medium. The latter of them is density of the mass medium [2]. 
le 2. The spread velocity of sound in from the various substances

\begin{tabular}{|c|c|c|}
\hline Substance & $\begin{array}{c}\text { Density } \\
\text { Air }\end{array}$ & Velocity \\
\hline Oil & 0,001 & 331 \\
\hline Water & 1,93 & 1450 \\
\hline Bone & 1,85 & 1540 \\
\hline Aluminum & 2,7 & 4080 \\
\hline
\end{tabular}

\subsection{Application Area of Ultrasound}

The use of industry;

- To bring metals into thin powder

- To prepare emulsions of very fine-grained photo

- To make custom metal alloys

- To separate gases, gas mixtures

- To prevent contamination of the environment by cleaning air gases from factory chimneys

- Textile, metal coating, watchmaker, to make the cleaning process, such as requiring extreme cleanliness

- To make ultrasonic drilling machine for hard metals drilling and the processing

- Foundry, to purify molten metal from gases and to control crystal growth

- To use sonar device on submarine and ships

The use of medicine;

Ultrasound devices, which are commonly used in the medicine, are the most commonly used diagnostic's method [3].

\subsection{Piezoelectric Effect}

The electromechanical generators can be magnetostrictive or piezoelectric. The feeder of the piezoelectric generator converts electrical energy of $50 / 60 \mathrm{~Hz}$ into electrical energy with high frequency. This energy is transmitted to the piezoelectric transducer which is converted into mechanical vibrations. When the electrical energy applied to upon piezoelectric material, the mechanical vibrations occur between the ends of crystal. At the same time the vibration creates the wave of ultrasound wave. Through the propagation of this wave, compression and rebound action occur, where the pressure is positive, negative, respectively. In areas with negative pressure, vapor bubbles can be formed. Cavitation phenomenon is produced due to intense local pressure because of cavities extinction and implosions.
This occurs during compression, when positive pre annihilate cavity. During cavities implosion, particularly those near the liquid surface are generated by intense hydraulic shocks. These shocks initiate disintegration of liquid film and production of droplets.

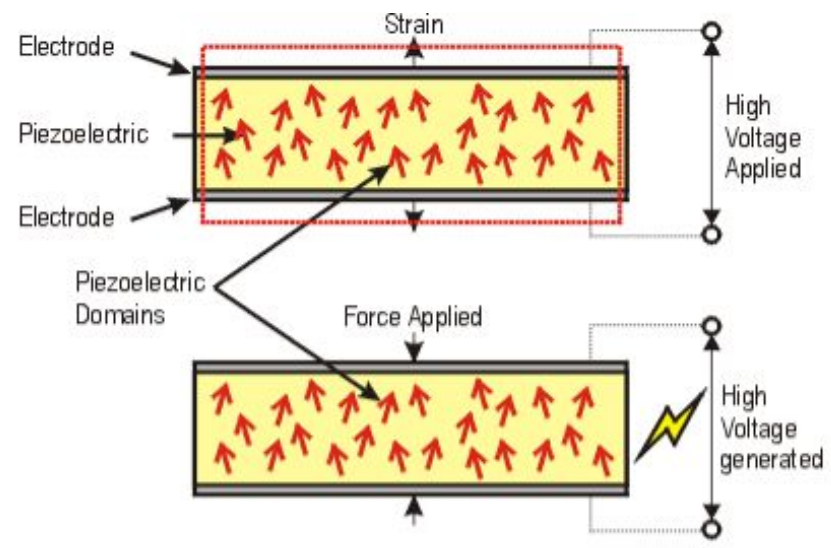

Figure 2. Piezoelectric Effect

\subsection{Ultrasonic Nebulization}

In the ultrasonic nebulizer used to create vapor are used piezoelectric crystal which is made vibration higher than 1 MHz's. Crystal transducer is made from materials such as quartz-barium, titanium, converts electrical signal to sound. Bunch of sound, which is focused in the liquid, generates waves. When the frequency and amplitude is strong enough, wave crest, which is like umbrella, reaches surface of liquid. The ultrasonic sound spread in the liquid is given an energy and act to the molecules of liquid. So it begins rupture the surface of liquid and liquid is converted the fog.

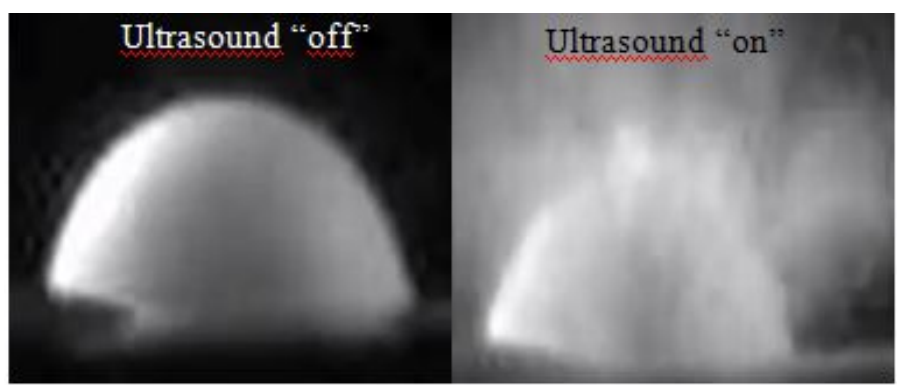

Figure.3 Ultrasonic sound effect on a water droplet

\section{Material and methods}

In this study of used method is experimental study. The experiment mechanism was founded for ultrasonic atomization. At the same time a camera system was founded for measurement droplet size. During operation, the finding of experiment was transmitted in the computer. Image taken from was analyzed the image processing program. On the other hand, a conventional injector which is used internal combustion engine that it is called MPI injection system was investigated under the similar conditionals. 
ary Iils comparison was made for to see between alfierences of atomization methods.

\subsection{Experimental Mechanism}

Pro The main purpose of this experiment is to compare the droplet size generated by ultrasonic sound and conventional gasoline injector. vide sufficient detail to allow the work to be reproduced. Methods already published should be indicated by a reference: only relevant modifications should be described.

\subsubsection{Gasoline Direct Injection System}

In this experiment was compared gasoline direct injection system because in the spark ignition engine latest injection technology is this technology. It is more efficient than other gasoline injection systems. Atomization is better. Therefore, gasoline direct injection system that it is in the literature was compared with ultrasonic atomization.

At the article, was made by L. Alloca and et al. in 2009, they were prepared as follows an experiment mechanism.

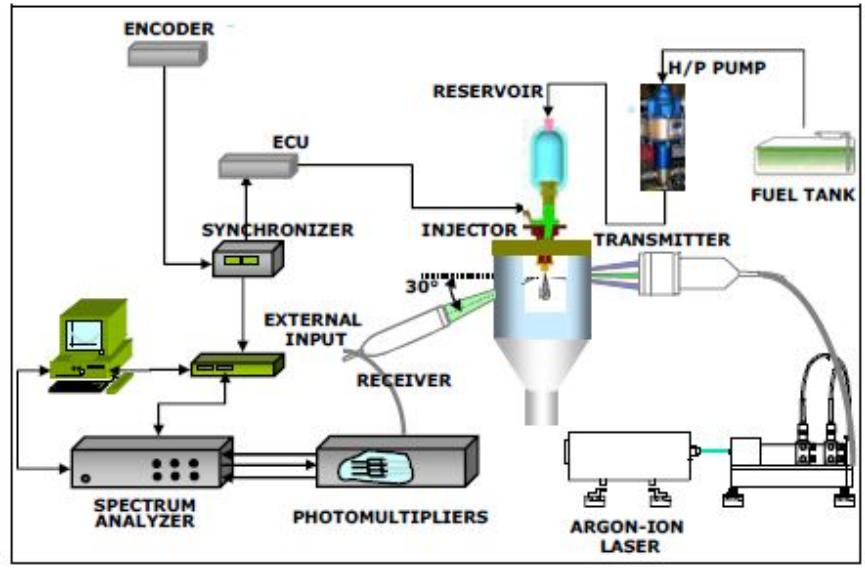

Figure. 4 The experiment Mechanism set up by L. Alloca and et al.[4].

As a result of this study, at the gasoline direct injection system was occurred droplet size as following shows graphic.

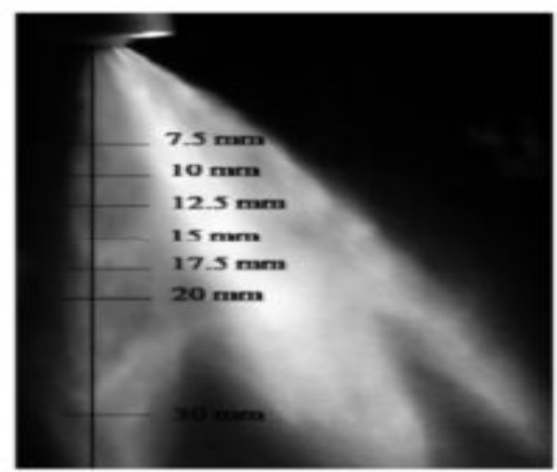

Figure.5 Measurement grid

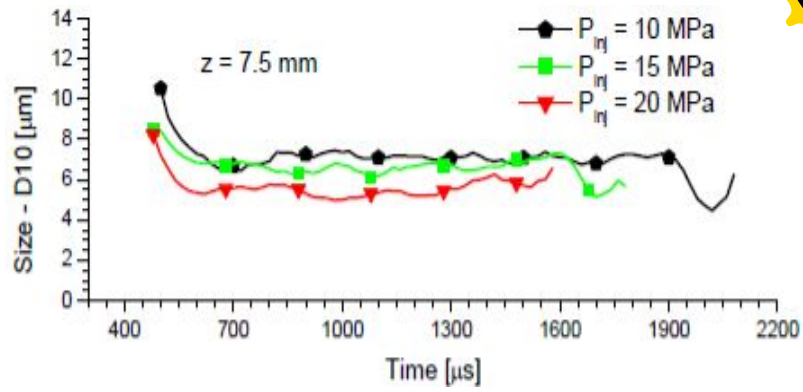

Figure 6 The investigated points have been $7.5 \mathrm{~mm}$ from the nozzle

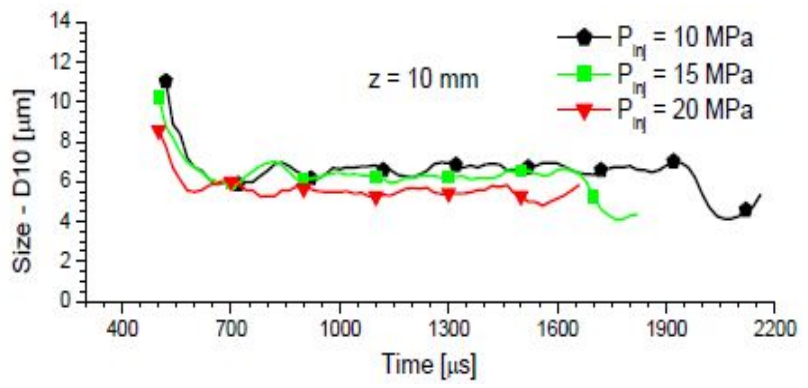

Figure 7 The investigated points have been $10 \mathrm{~mm}$ from the nozzle

Shows the above graphs changed between 6 to $12 \mu \mathrm{m}$ of droplet size at the gasoline direct injection.

\subsubsection{The Ultrasonic Atomization System}

In this system the most important advantage is no need the pressure. So without pressure is made the better atomization. Schematic view of the experiment mechanism of ultrasonic atomization as follows.

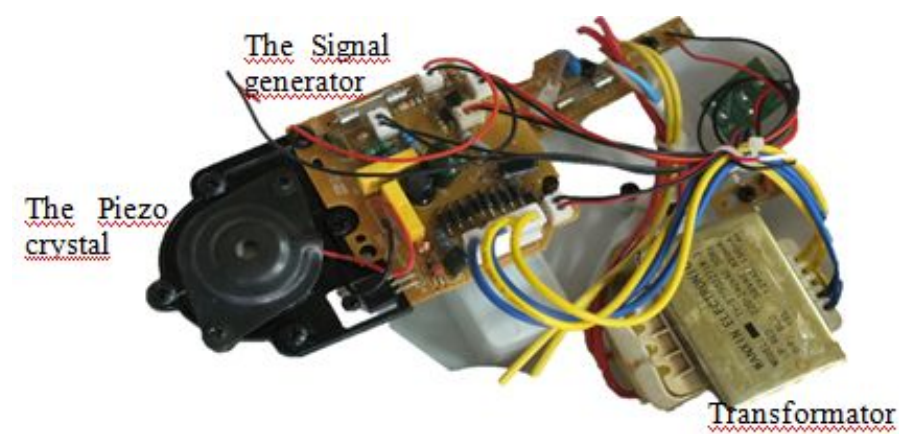

Figur8 The experimental mechanism of ultrasonic generator.

In this mechanism, the electric voltage reduced from $220 \mathrm{~V}$ to $12-40 \mathrm{~V}$ by the transformator. The signal generator is produces the necessary signal for contraction and relaxation of piezo crystal. When this signals goes on piezo crystal, ultrasonic sound is produced. That the ultrasonic sound wave sent on the liquid, it is begun atomization. 
